\title{
0277 ASSESSING INJURY EMERGENCY RESPONSE OF VILLAGE HEALTH WORKERS AND VOLUNTEERS IN FIVE PROVINCES IN VIETNAM
}

T H T Nguyen*, L T T Ngoc, H T L Nguyen, M A Luong Correspondence: Department of Preventive Medicine, Ministry of Health, Unit 3, Floor 11, HAREC Buiding, 4A LAng Ha street Hanoi 10000, Vietnam

10.1136/ip.2010.029215.277

In Vietnam, road traffic injury is the leading cause of injuries with about 12000 death cases per year. The prehospital care is still a new issue in the injury prevention work. Village health workers (VHWs) and health volunteers are considered as first tiers of prehospital care in Vietnam. Therefore, it is necessary to assess the injury emergency response capacity of village health workers and volunteers in order to strengthen their capacity.

Methodology Cross sectional and qualitative methods including interview of $3000 \mathrm{VHWs}$ and volunteers in $60 \mathrm{com}$ munes in Vietnam.

Result Nearly $70 \%$ of volunteer and VHW graduated from middle school and high school. In 60 communes, the main cause of injury mortality during 2007-2008 was road traffic $64.5 \%$. There were three death cases due to RTA in each commune in average. The VHW and volunteers provided prehospital care for $10 \%$ of road traffic injury (equal about 400 cases)

per year in all cities/provinces. Only $16 \%$ of volunteers are equipped with essential tools for providing injuries and accident emergency care. $74.5 \%$ of volunteers and VHW had seen traffic accidents. $36.7 \%$ of volunteers had experiences with traffic injury emergency care. $22 \%$ of volunteers/VHW had reported on injuries and accidents; $31.2 \%$ of volunteers/VHW had participated in training courses for injuries emergency care and $37.7 \%$ of them had got training from local hospitals.

Conclusion Volunteers and VHW should be provided training, essential emergency trauma care equipments for road traffic injuries. 